\title{
INVERSE PRODUCING EXTENSION OF A BANACH ALGEBRA WHICH ELIMINATES THE RESIDUAL SPECTRUM OF ONE ELEMENT
}

\author{
BY
}

C. J. READ

\begin{abstract}
If $A$ is a commutative unital Banach algebra and $G \subset A$ is a collection of nontopological zero divisors, the question arises whether we can find an extension $A^{\prime}$ of $A$ in which every element of $G$ has an inverse. Shilov [1] proved that this was the case if $G$ consisted of a single element, and Arens [2] conjectures that it might be true for any set $G$. In [3], Bollobás proved that this is not the case, and gave an example of an uncountable set $G$ for which no extension $A^{\prime}$ can contain inverses for more than countably many elements of $G$. Bollobás proved that it was possible to find inverses for any countable $G$, and gave best possible bounds for the norms of the inverses in [4].

In this paper, it is proved that inverses can always be found if the elements of $G$ differ only by multiples of the unit; that is, we can eliminate the residual spectrum of one element of $A$. This answers the question posed by Bollobás in [5].
\end{abstract}

1. Preliminary definitions and statement of the main result. Throughout this paper, a Banach algebra is assumed to be commutative and to possess a unit.

If $A$ is a Banach algebra, $x \in A$, then the essential spectrum of $x$ in $A$ is the set

$$
\sigma_{e}(x)=\left\{\lambda \in \mathrm{C}: \lambda \cdot \mathbf{1}_{A}-x \text { is a topological zero divisor }\right\},
$$

and the residual spectrum of $x$ in $A$ is the set

$\sigma_{r}(x)=\left\{\lambda \in \mathrm{C}: \lambda \cdot \mathbf{1}_{\boldsymbol{A}}-x\right.$ is not invertible, but is not a

topological zero divisor\}.

Thus our main theorem may be stated as follows.

THEOREM 1. Let $A$ be a commutative Banach algebra, $x \in A$. Then there is an extension $A^{\prime}$ of $A$ in which the spectrum of $x$ is precisely the essential spectrum of $x$ in $A$. 2.

Before proving Theorem 1, we prove the weaker result stated here as Theorem

THEOREM 2. Let $A$ be a Banach algebra, $x \in A$, and let $K$ be a compact set in the residual spectrum of $x$ in $A$. Then there is an extension $A^{\prime}$ of $A$, such that the spectrum of $x$ in $A^{\prime}$ does not intersect $K$.

The method used to prove Theorem 2 is to take an open neighbourhood $U$ of the essential spectrum of $x$ in $A$, whose closure does not intersect $K$. (Such a

Received by the editors November 24, 1981 and, in revised form, November 15, 1983.

1980 Mathematics Subject Clasrification. Primary 46J20; Secondary 47D30, 32D15.

C) 1984 American Mathematical Society $0002-9947 / 84 \$ 1.00+\$ .25$ per page 
neighbourhood can always be found since the essential spectrum and the set $K$ are two nonintersecting compact sets in C.) We then consider the algebra $X$ of bounded analytic maps $U \rightarrow A$, and give it the supremum norm; this is a Banach algebra in which $A$ is embedded isometrically as the constant functions.

We then let $J \subset X$ denote the closed ideal generated by the function $h \in X$, where

$$
\begin{aligned}
h: & U \rightarrow A \\
& : \lambda \rightarrow \lambda \cdot 1_{A}-x
\end{aligned}
$$

(we shall see that the ideal generated by this function is closed anyway).

We shall find that the algebra $A$ is embedded (up to isomorphism) in the quotient space $X / J$. But then $X / J$ is an extension of $A$, and the spectrum of $x$ when embedded in $X / J$ does not intersect $K$.

To see this, let $\mu \in K$, and let us find an inverse for $\mu \cdot 1_{A}-x$ when embedded in $X / J$. Let

$$
\begin{aligned}
R_{\mu}: & U \rightarrow A \\
: & \lambda \rightarrow(\mu-\lambda)^{-1} \cdot \mathbf{1}_{A} .
\end{aligned}
$$

This is a bounded analytic map $U \rightarrow A$ (since $K$ and $U$ are a finite distance apart). So $R_{\mu} \in X$. Also, the element $\mu \cdot 1_{A}-x$ is embedded in $X$ as the constant function

$$
\begin{aligned}
c_{\mu}: & U \rightarrow A \\
: & \lambda \rightarrow \mu \cdot 1_{A}-x .
\end{aligned}
$$

Let $q: X \rightarrow X / J$ denote the quotient map. Then,

$$
\begin{aligned}
R_{\mu} \cdot c_{\mu}: & U \rightarrow A \\
: & \lambda \rightarrow\left((\mu-\lambda)^{-1} \cdot 1_{A}\right) \cdot\left(\mu \cdot 1_{A}-x\right) \\
& =(\mu-\lambda)^{-1} \cdot 1_{A} \cdot\left((\mu-\lambda) \cdot 1_{A}+\left(\lambda \cdot 1_{A}-x\right)\right) \\
& =1_{A}+(\mu-\lambda)^{-1} \cdot\left(\lambda \cdot 1_{A}-x\right) .
\end{aligned}
$$

Thus

where $h: \lambda \rightarrow(\mu-\lambda)^{-1} \cdot\left(\lambda \cdot 1_{A}-x\right)$ and

$$
q\left(R_{\mu}\right) \cdot q\left(c_{\mu}\right)=q\left(1_{x}\right)+q(h)
$$

$$
\begin{aligned}
1_{x}: U & \rightarrow A \\
: & \lambda \rightarrow 1_{A} .
\end{aligned}
$$

But $h$ is in the ideal $J$, so $q(h)=0$. Thus $q\left(R_{\mu}\right) \cdot q\left(c_{\mu}\right)=q\left(1_{x}\right)$, so the element $\mu \cdot 1_{A}-x \in A$ has an inverse $q\left(R_{\mu}\right)$, when embedded as $q\left(c_{\mu}\right) \in X / J$.

Thus Theorem 2 will be proved.

An important tool in proving Theorem 2 and, later, Theorem 1, is the following

LEMMA 3. Let $B$ be a Banach algebra, $x \in B$, and let $U$ and $V$ be open sets in C such that

(1) $U$ contains the essential spectrum of $x$ in $B$,

(2) $V$ contains $U$, and

(3) every component of $V$ intersects $U$.

Suppose we have $f(\lambda)=(\lambda-x) g(\lambda)$ (all $\lambda \in U)$, where $f$ and $g$ are analytic functions $f: V \rightarrow B$ and $g: U \rightarrow B$. 
Then there is an analytic extension $g: V \rightarrow B$ of $g$.

ProOF OF LEMMA 3. We can find an open set $W \subset \mathrm{C}$, such that $V=U \cup W$ and $\bar{W} \cap \sigma_{e}(x)=\varnothing$. Each component of $W$ will intersect $U$, and our problem is to extend the analytic germs of $g$ from $U \cap W$ to all of $W$.

Since

$$
\bar{W} \cap \sigma_{e}(x)=\varnothing,
$$

we claim that there is an $\varepsilon>0$ such that $\lambda \in W, a \in A$ implies

$$
\left\|\left(\lambda \cdot 1_{A}-x\right) \cdot a\right\| \geq \varepsilon\|a\| .
$$

For if not, there are sequences $\left(\lambda_{n}\right)_{n=1}^{\infty} \subset W$, and $\left(a_{n}\right)_{n=1}^{\infty} \subset A$, with each $\left\|a_{n}\right\|=1$, and

$$
\left\|\left(\lambda_{n} \cdot 1_{A}-x\right) \cdot a_{n}\right\| \rightarrow 0 \text { as } n \rightarrow \infty .
$$

Then $\left\{\left|\lambda_{n}\right|: n \in \mathbf{N}\right\}$ must be bounded, so we may assume (taking a subsequence if necessary) that $\lambda_{n} \rightarrow \lambda \in \bar{W}$. Then

$$
\begin{aligned}
\left\|\left(\lambda \cdot 1_{A}-x\right) \cdot a_{n}\right\| & \leq\left\|\left(\lambda_{n} \cdot 1_{A}-x\right) a_{n}\right\|+\left\|\left(\lambda-\lambda_{n}\right) a_{n}\right\| \\
& =\left\|\left(\lambda_{n} \cdot 1_{A}-x\right) a_{n}\right\|+\left|\lambda-\lambda_{n}\right| \\
& \rightarrow 0 \text { as } n \rightarrow \infty .
\end{aligned}
$$

Thus $\lambda \cdot 1_{A}-x$ is a topological zero divisor in $A$, so $\lambda \in \bar{W} \cap \sigma_{e}(x)$, contradicting our observation (*) that this set is empty. Let us choose an $\varepsilon>0$ such that condition (3.1) is satisfied.

Now,

$$
f(\lambda)=\left(\lambda \cdot 1_{A}-x\right) \cdot g(\lambda)
$$

which implies that, for each $n=1,2, \ldots$,

$$
f^{(n)}(\lambda)=\left(\lambda \cdot 1_{A}-x\right) \cdot g^{(n)}(\lambda)+n g^{(n-1)}(\lambda),
$$

where $h^{(r)}$ denotes the $r$ th derivative of a function $h$.

It follows that, if we have an analytic germ of $g$ at some point $\lambda_{0} \in W$, then

$$
\left\|g^{(n)}\left(\lambda_{0}\right)\right\| \leq \sum_{r=0}^{n}\left\|f^{(r)}\left(\lambda_{0}\right)\right\| \cdot \frac{n !}{r !} \cdot\left(\frac{1}{\varepsilon}\right)^{n-r}
$$

for all $n \in \mathbf{N}$.

So if the power series for $f$ at $\lambda_{0}$ has radius of convergence $\delta>0$, then the power series for $g$ at $\lambda_{0}$ has radius of convergence greater than or equal to $\varepsilon \cdot \delta$.

Therefore this radius of convergence is bounded away from zero on any compact set in $W$; hence it must be possible to extend $g$ throughout $W$, as required. Thus Lemma 3 is proved.

COROllary 4. Let $B$ be a Banach algebra, $c \in B$, and suppose that

$$
c=\left(\lambda \cdot 1_{A}-x\right) \cdot g(\lambda) \quad(\text { all } \lambda \in U),
$$

where $g: U \rightarrow B$ is analytic, and $U$ is a neighbourhood of the essential spectrum of $c$ in B. Then $c=0$.

ProOF. The constant function $c$ can be extended to all of $C$, hence, by Lemma 3 , the function $g$ extends to all of $C$, and the extension is a bounded entire function, 
which must be constant. Thus we have $c=\left(\lambda \cdot 1_{A}-x\right) \cdot g$ (all $\lambda \in \mathrm{C}$ ) for some $g \in A$; therefore $c=g=0$.

We now prove Theorem 2 .

Proof OF THEOREM 2. We are given a Banach algebra $A$, an element $x \in A$, and a compact set $K$ in the residual spectrum of $X$ in $A$. We wish to exhibit an extension $A^{\prime}$ of $A$, such that the spectrum of $x$ in $A$ does not intersect $K$.

Let us choose a bounded open set $U \subset \mathbf{C}$ such that $U \supset \sigma_{e}(x)$ and $\bar{U} \cap K=\varnothing$. Let $X$ be the Banach algebra of bounded analytic functions $U \rightarrow A$, with the supremum norm,

$$
\|f: U \rightarrow A\|_{X}=\sup _{\lambda \in U}\|f(\lambda)\|_{A} .
$$

(Note: we do not demand that such a function have a continuous extension to $\bar{U}$; this is important when we come to prove Theorem 1.) Let $J$ be the closed ideal in $X$ generated by the function $h \in X$, where $h(\lambda)=\lambda \cdot 1_{A}-x($ all $\lambda \in U)$.

Consider the isometric embedding $j: A \rightarrow X$ sending $c \in A$ to the constant function $j(c): \lambda \rightarrow c($ all $\lambda \in U)$.

We wish to show that the morphism $\psi: A \rightarrow X / J$ obtained by composing $j$ and the quotient map $q: X \rightarrow X / J$, is still an isomorphism.

Now it is evident that

$$
\|\psi(a)\| \leq\|a\| \text { for all } a \in A
$$

so we need to check that there is no sequence $\left(c_{i}\right)_{i=1}^{\infty} \subset A$, such that each $\left\|c_{i}\right\|=1$, but

$$
\left\|\psi\left(c_{i}\right)\right\|_{X / J} \rightarrow 0 \text { as } i \rightarrow \infty \text {. }
$$

Now $J$ is the closure of the set of all functions $H \in X$ of form

$$
\begin{aligned}
H: & U \rightarrow A \\
& : \lambda \rightarrow f(\lambda) \cdot\left(\lambda \cdot 1_{A}-x\right),
\end{aligned}
$$

where $f \in X$. Thus if $\left\|\psi\left(c_{i}\right)\right\|_{X / J} \rightarrow 0$ as $i \rightarrow \infty$, there must be functions $\left(f_{i}\right)_{i=1}^{\infty} \subset$ $X$ such that

$$
\sup _{\lambda \in U}\left\|f_{i}(\lambda) \cdot\left(\lambda \cdot 1_{A}-x\right)-c_{i}\right\|_{A} \rightarrow 0 \text { as } i \rightarrow \infty \text {. }
$$

We have to show that such constants and functions cannot exist. We state this as a separate lemma:

LEMMA 2.1. If $A$ is a Banach algebra, $x \in A$, and $U \subset \mathbf{C}$ is an open set containing the essential spectrum of $x$ in $A$, then there is an $\varepsilon>0$ such that for all $c \in A$, and all analytic functions $f: U \rightarrow \mathbf{C}$, we have

$$
\sup _{\lambda \in U}\left\|f(\lambda) \cdot\left(\lambda \cdot 1_{A}-x\right)-c\right\|_{A} \geq \varepsilon\|c\| .
$$

PROOF OF LEMMA 2.1. Let $B$ be the Banach algebra of bounded sequences $\left(a_{i}\right)_{i=1}^{\infty} \subset A$, with pointwise addition and multiplication, and the norm

$$
\left\|\left(a_{i}\right)_{i=1}^{\infty}\right\|_{B}=\sup _{i \in \mathbf{N}}\left\|a_{i}\right\|_{A}
$$

Let $I$ be the closed ideal of $B$ consisting of those sequences in $B$ which norm converge to zero. Let $\pi$ be the natural projection $\pi: B \rightarrow B / I$. 
Now, for all $\left(a_{i}\right)_{i=1}^{\infty} \in B$,

$$
\left\|\pi\left(\left(a_{i}\right)_{i=1}^{\infty}\right)\right\|_{B / I}=\limsup _{i \rightarrow \infty}\left\|a_{i}\right\|_{A}
$$

and $B / I$ is a commutative Banach algebra with unit $1_{B / I}=\pi\left[\left(1_{A}, 1_{A}, 1_{A}, \ldots\right)\right]$ (the equivalence class of the sequence in $B$ consisting entirely of 1 's).

$A$ is embedded in $B / I$ by the isometry

$$
\begin{aligned}
\phi: & A \rightarrow B / I \\
: & a \rightarrow \pi[(a, a, a, \ldots)] .
\end{aligned}
$$

In fact, our element $x$ will have exactly the same spectrum and essential spectrum as $\phi(x) \in B / I$ as it did in $A$.

Suppose our lemma is false. Let $\left(c_{i}\right)_{i=1}^{\infty}$ be a sequence of norm 1 elements of $A$, and $\left(f_{i}\right)_{i=1}^{\infty}$ a sequence of analytic functions $U \rightarrow A$, such that

$$
\sup _{\lambda \in U}\left\|f_{i}(\lambda) \cdot\left(\lambda \cdot 1_{A}-x\right)-c_{i}\right\| \rightarrow 0 \quad \text { as } i \rightarrow \infty .
$$

Now if $W \subset U$ is any set which is bounded away from the essential spectrum of $x$, then there is an $\eta>0$ such that

$$
\left\|a \cdot\left(\lambda \cdot 1_{A}-x\right)\right\| \geq \eta\|a\| \text { for all } a \in A, \lambda \in W .
$$

Therefore for all $i \in \mathbf{N}, \lambda \in W$ we have

$$
\left\|f_{i}(\lambda) \cdot\left(\lambda \cdot 1_{A}-x\right)-c_{i}\right\| \geq \eta\left\|f_{i}(\lambda)\right\|-\left\|c_{i}\right\|=\eta\left\|f_{i}(\lambda)\right\|-1 .
$$

Thus there is a uniform bound on the values $\left\|f_{i}(\lambda)\right\|$ for all $i$, throughout $W$.

However, since the essential spectrum is a compact set within $U$, we may pick a suitable set $W$ so that, by the Maximum Modulus principle, each $f_{i}$ approaches its supremum norm $\sup _{\lambda \in U}\left\|f_{i}(\lambda)\right\|$ somewhere on $W$ (we must say "approaches" rather than "achieves" since $U$ is an open set so the supremum need not be achieved anywhere).

It follows that the collection $\left(f_{i}\right)_{i=1}^{\infty}$ is uniformly norm bounded. Similarly, the collection is uniformly differentiable at any point $u \in U$.

Now consider the map

$$
\begin{aligned}
F: & U \rightarrow B / I \\
& : \lambda \rightarrow \pi\left[\left(f_{1}(\lambda), f_{2}(\lambda), f_{3}(\lambda), \ldots\right)\right] .
\end{aligned}
$$

Since the $f_{i}$ 's are uniformly bounded, the sequence $\left(f_{1}(\lambda), f_{2}(\lambda), f_{3}(\lambda), \ldots\right)$ is always in $B$; and since they are uniformly differentiable, we find that $F$ is a uniformly bounded analytic map. It is easy to see that for all $\lambda \in U$,

$$
\left(\lambda \cdot 1_{B / I}-\phi(x)\right) \cdot F(\lambda)=\pi\left[\left(c_{1}, c_{2}, c_{3}, \ldots\right)\right] .
$$

Therefore, since $U$ contains the essential spectrum of $\phi(x) \in B / I$, we have by Corollary 4 that $\pi\left[\left(c_{1}, c_{2}, c_{3}, \ldots\right)\right]=0$. Therefore $\lim \sup _{i \rightarrow \infty}\left\|c_{i}\right\|_{A}=0$, but this is a contradiction since by hypothesis each $\left\|c_{i}\right\|=1$. Thus Lemma 2.1 is proved. 
COROllary 2.2. The map $\psi: A \rightarrow X / J$ is an isomorphism.

By the result of $[6]$, we can put an equivalent norm on $X / J$ so that $A$ is now embedded isometrically by the map $\psi$. But this proves the theorem, for $X / J$ contains an inverse to $\mu \cdot 1_{A}-x$ for every $\mu$ in $K$, namely the element $q\left(R_{\mu}\right)$, where

$$
\begin{aligned}
R_{\mu}: & U \rightarrow A \\
& : \lambda \rightarrow(\mu-\lambda)^{-1} \cdot 1_{A} .
\end{aligned}
$$

Having proved Theorem 2, we now make some definitions which lead towards a proof of Theorem 1 .

Let $\Omega$ denote the collection of all countable ordinals. With each ordinal $\alpha \in \Omega$, we shall associate a Banach algebra $A(\alpha)$; the collection $\{A(\alpha): \alpha \in \Omega\}$ will be directed upwards, in the sense that for all $\alpha, \beta \in \Omega, \alpha<\beta$, there will be an isometric embedding

$$
\tau_{\alpha, \beta}: A(\alpha) \rightarrow A(\beta)
$$

Furthermore, if $\alpha, \beta, \gamma \in \Omega(\alpha<\beta<\gamma)$, we will have $\tau_{\beta, \gamma} \circ \tau_{\alpha, \beta}=\tau_{\alpha, \gamma}$.

We now proceed to define our sequence of Banach algebras, using transfinite induction.

(1) We define $A(1)$ to be our original Banach algebra $A$.

(2) Given $\alpha \in \Omega$ and the Banach algebra $A(\alpha)$, we define $A(\alpha+1)$ as follows:

Let $B(\alpha)$ be the Banach algebra of sequences $\left(a_{i}\right)_{i=1}^{\infty}, a_{i} \in A(\alpha)$, with pointwise addition and multiplication and the supremum norm

$$
\left\|\left(a_{i}\right)_{i=1}^{\infty}\right\|_{B(\alpha)}=\sup _{i \in \mathbf{N}}\left\|a_{i}\right\|_{A(\alpha)}
$$

let $I(\alpha)$ be the closed ideal consisting of all sequences in $B(\alpha)$ which norm converge to zero, and let $\pi_{\alpha}$ denote the natural projection

$$
\pi_{\alpha}: B(\alpha) \rightarrow B(\alpha) / I(\alpha) .
$$

We define $A(\alpha+1)=B(\alpha) / I(\alpha)$, and the map $\tau_{\alpha, \alpha+1}$ is the isometric embedding

$$
\begin{aligned}
j_{\alpha}: & A(\alpha) \rightarrow A(\alpha+1) \\
: & a \rightarrow \pi_{\alpha}[(a, a, a, a, \ldots)] .
\end{aligned}
$$

We mist then define $\tau_{\beta, \alpha+1}=j_{\alpha} \circ \tau_{\beta, \alpha}$ for each ordinal $\beta<\alpha$.

(3) If $\alpha_{i} \in \Omega, i=1,2, \ldots$, then we require that $A\left(\bigcup_{i} \alpha_{i}\right)$ be the completion of the direct limit of the collection $\left\{A\left(\alpha_{i}\right), i=1,2,3, \ldots\right\}$ of Banach algebras, which is directed by the maps $\tau_{\alpha_{i}, \alpha_{j}}\left(\alpha_{i}<\alpha_{j}\right)$. For $\beta<\bigcup_{i} \alpha_{i}$, the map

$$
\tau_{\beta, \cup, \alpha_{i}}: A(\beta) \rightarrow A\left(\bigcup_{i} \alpha_{i}\right)
$$

is the direct limit of the maps $\tau_{\beta, \alpha_{i}}: \beta<\alpha_{i}$, followed by the map which sends the direct limit of the algebras $A\left(\alpha_{i}\right)$ to its completion, which is $A\left(\bigcup_{i} \alpha_{i}\right)$.

Now these three conditions define the collection $\{A(\alpha), \alpha \in \Omega\}$ uniquely, together with the linking maps $\tau_{\beta, \alpha}: \beta<\alpha$. 
LeMmA 5. If $\alpha, \beta \in \Omega(\alpha \leq \beta)$ and $a \in A(\alpha)$, then

$$
\inf _{y \in A(\beta)}\left(\frac{\left\|\tau_{\alpha, \beta}(a) \cdot y\right\|}{\|y\|}\right)=\inf _{y_{0} \in A(\alpha)}\left(\frac{\left\|a y_{0}\right\|}{\left\|y_{0}\right\|}\right) .
$$

PROOF. The left-hand side of this identity is an infimum similar to that on the right-hand side, but evaluated with the element $a \in A(\alpha)$ embedded in a larger space $A(\beta)$. It is therefore less than or equal to the right-hand side.

The opposite inequality is proved by transfinite induction on $\beta$; the result is trivially true if $\beta=\alpha$.

If the result is true for $\beta=\beta_{0}$, then given $y \in A\left(\beta_{0}+1\right)$ let us say

$$
y=\pi_{\alpha}\left[\left(y_{i}\right)_{i=1}^{\infty}\right] \quad\left(y_{i} \in A\left(\beta_{0}\right)\right)
$$

then

$$
\begin{aligned}
\left\|y \cdot \tau_{\alpha, \beta_{0}+1}(a)\right\|_{A\left(\beta_{0}+1\right)} & =\limsup _{i \rightarrow \infty}\left(\left\|y_{i} \cdot \tau_{\alpha, \beta_{0}}(a)\right\|_{A\left(\beta_{0}\right)}\right) \\
& \geq \limsup _{i \rightarrow \infty}\left\|y_{i}\right\| \cdot \inf _{y^{\prime} \in A\left(\beta_{0}\right)}\left(\frac{\left\|y^{\prime} \cdot \tau_{\alpha, \beta_{0}}(a)\right\|}{\left\|y^{\prime}\right\|}\right) \\
& \geq \limsup _{i \rightarrow \infty}\left\|y_{i}\right\| \cdot \inf _{y_{0} \in A(\alpha)}\left(\frac{\left\|y_{0} a\right\|}{\left\|y_{0}\right\|}\right)
\end{aligned}
$$

(by induction hypothesis)

$$
=\|y\|_{A\left(\beta_{0}+1\right)} \cdot \inf _{y_{0} \in A(\alpha)}\left(\frac{\left\|y_{0} a\right\|}{\left\|y_{0}\right\|}\right) \text {. }
$$

This is the result for $\beta_{0}+1$. But it is clear that an equality such as $(*)$ is preserved under direct limits and completions. So the result is true for all $\beta \in \Omega$.

We now prove Theorem 1.

ProOF OF THEOREM 1. Given a Banach algebra $A$ and $x \in A$, let us choose a sequence $\left(U_{i}\right)_{i=1}^{\infty}$ of bounded open sets in $C$, such that:

(1) For each $i, U_{i} \supset U_{i+1}$.

(2) For each $i$, every component of $U_{i}$ intersects $U_{i+1}$.

(3) $\bigcap_{i=1}^{\infty} U_{i}=\sigma_{e}(x)$.

DEFINITION. A sequence $\left(\varepsilon_{i}\right)_{i=1}^{n}$ of strictly positive real numbers is said to be "admissible" for $\alpha \in \Omega$ if, whenever there are bounded analytic functions

$$
g_{i}: U_{i} \rightarrow A(\alpha) \quad(i=1,2, \ldots, n),
$$

an analytic function $f: U_{n} \rightarrow A(\alpha)$ and a constant $c \in A(\alpha)$ such that, for all $\lambda \in U_{n}$,

$$
c=\sum_{i=1}^{n} g_{i}(\lambda)+\tau_{1, \alpha}\left(\lambda \cdot 1_{A}-x\right) \cdot f(\lambda)
$$

then

$$
\|c\|_{A(\alpha)} \leq \sum_{i=1}^{n} \varepsilon_{i}^{-1} \cdot \sup _{\lambda \in U_{i}}\left\|g_{i}(\lambda)\right\|_{A(\alpha)} .
$$


The most important step in proving Theorem 1 is the following

LEMMA 6 . There is a sequence $\left(\varepsilon_{i}\right)_{i=1}^{\infty}$ such that, for each $n$, the sequence $\left(\varepsilon_{i}\right)_{i=1}^{n}$ is admissible for every $\alpha \in \Omega$.

PROOF OF LEMMA 6. It is enough to show the following:

(1) There is an $\varepsilon_{1}$, which is admissible, as a sequence of length 1 , for every $\alpha \in \Omega$.

(2) If the sequence $\left(\varepsilon_{i}\right)_{i=1}^{n}$ is admissible for every $\alpha \in \Omega$ and $\delta>0$, then there is an $\varepsilon_{n+1}>0$ such that the sequence

$$
(1+\delta)^{-1} \cdot \varepsilon_{1},(1+\delta)^{-1} \cdot \varepsilon_{2}, \ldots,(1+\delta)^{-1} \cdot \varepsilon_{n}, \varepsilon_{n+1}
$$

is also admissible for every $\alpha \in \Omega$.

Let us apply Lemma 2.1 with $A$ replaced by $A(\alpha)$ for some ordinal $\alpha, x$ replaced by $\tau_{1, \alpha}(x)$ and $U$ replaced by $U_{1}$. We find that there is an $\varepsilon>0$ such that, for all $c \in A(\alpha)$ and all analytic functions $f: U_{1} \rightarrow A(\alpha)$, we have

$$
\sup _{\lambda \in U_{1}}\left\|f(\lambda) \cdot\left(\lambda \cdot 1_{A}-x\right)-c\right\|_{A(\alpha)} \geq \varepsilon\|c\| .
$$

Writing $g_{1}(\lambda)=c-f(\lambda) \cdot\left(\lambda \cdot 1_{A}-x\right)\left(\lambda \in U_{1}\right)$ we see the value $\varepsilon$, a "sequence" of length 1 , is admissible for $\alpha$.

Denote by $\varepsilon_{1}(\alpha)$ the supremum of all admissible values of $\varepsilon$ for a given $\alpha$. This decreases with increasing $\alpha$, since the Banach algebras involved are always getting larger.

But $\varepsilon_{1}(\alpha)$ must be bounded away from zero. For if we could find a sequence $\left(\alpha_{i}\right)_{i=1}^{\infty}$ such that $\alpha_{i} \in \Omega$ and $\varepsilon_{1}\left(\alpha_{i}\right) \rightarrow 0$ as $i \rightarrow \infty$, then we must have $\varepsilon_{1}\left(\bigcup_{i=1}^{\infty} \alpha_{i}\right)$ $=0$, which is impossible.

Thus there is an $\varepsilon_{1}$ which is admissible for every $\alpha \in \Omega$. This proves assertion (1).

Now suppose that $\left(\varepsilon_{i}\right)_{i=1}^{n}$ is admissible for every $\alpha \in \Omega$. Choose a particular $\alpha \in \Omega$, and, given $\delta>0$, suppose that we cannot find an $\varepsilon_{n+1}$ such that

$$
(1+\delta)^{-1} \varepsilon_{1},(1+\delta)^{-1} \varepsilon_{2}, \ldots,(1+\delta)^{-1} \varepsilon_{n}, \varepsilon_{n+1}
$$

is admissible for $\alpha$.

Then we must be able to find constants $\left(c_{j}\right)_{j=1}^{\infty}$ in $A(\alpha)$, and analytic functions

$$
g_{i}^{(j)}: U_{i} \rightarrow A(\alpha) \quad(i=1,2, \ldots, n+1 ; j \in \mathbf{N})
$$

and

$$
f^{(j)}: U_{n+1} \rightarrow A(\alpha) \quad(j \in \mathbf{N})
$$

such that, for each $j,\left\|c_{j}\right\|=1$, and for all $\lambda \in U_{n+1}$,

$$
c_{j}=\sum_{i=1}^{n+1} g_{i}^{(j)}(\lambda)+\tau_{1, \alpha}\left(\lambda \cdot 1_{A}-x\right) \cdot f^{(j)}(\lambda),
$$

but

$$
1>(1+\delta) \sum_{i=1}^{n+1} \sup _{\lambda \in U_{i}}\left\|g_{i}^{(j)}(\lambda)\right\|+2^{j} \cdot \sup _{\lambda \in U_{n+1}}\left\|g_{n+1}^{(j)}(\lambda)\right\| .
$$

Extracting a subsequence from $\left(c_{k}\right)_{k=1}^{\infty}$, we may assume that

$$
\sup _{\lambda \in U_{2}}\left\|g_{i}^{(j)}(\lambda)\right\|_{\boldsymbol{A}(\alpha)} \rightarrow \eta_{i} \quad \text { as } j \rightarrow \infty, 1 \leq i \leq n
$$


where

$$
(1+\delta) \cdot \sum_{i=1}^{n} \varepsilon_{i}^{-1} \cdot \eta_{i} \leq 1
$$

But now, for each $i \leq n$ and $\lambda \in U_{i}$, define

$$
G_{i}(\lambda)=\pi_{a}\left[\left(g_{i}^{(1)}(\lambda), g_{i}^{(2)}(\lambda), \ldots, g_{i}^{(j)}(\lambda), \ldots\right)\right] .
$$

Define also, for $\lambda \in U_{n+1}$,

$$
F(\lambda)=\pi_{\alpha}\left[\left(f^{(1)}(\lambda), f^{(2)}(\lambda), \ldots, f^{(j)}(\lambda), \ldots\right)\right] \in A(\alpha+1) .
$$

These functions are analytic by arguments similar to those used in the proof of Theorem 2.

Then, for every $\lambda \in U_{n+1}$,

$$
\sum_{i=1}^{n} G_{i}(\lambda)+\left(\lambda \cdot 1_{A(\alpha+1)}-\tau_{1, \alpha+1}(x)\right) \cdot F(\lambda)=\pi_{\alpha}\left[\left(c_{1}, c_{2}, c_{3}, \ldots\right)\right] .
$$

Therefore, by Lemma 3 , we can extend $F$ to $U_{n}$ (this being the domain of definition of $\left.G_{n}\right)$, and so, since $\left(\varepsilon_{i}\right)_{i=1}^{n}$ is admissible for $\alpha+1$, we must have

$$
1=\left\|\pi_{\alpha}\left[\left(c_{1}, c_{2}, c_{3}, \ldots\right)\right]\right\| \leq \sum_{i=1}^{n} \sup _{\lambda \in U_{i}}\left\|G_{i}(\lambda)\right\| \cdot \varepsilon_{i}^{-1} .
$$

But $\sup _{\lambda \in U_{i}}\left\|G_{i}(\lambda)\right\| \leq \eta_{i}$ so, by $(*)$, we have a contradiction.

Thus, for each $\alpha \in \Omega$, there is a suitable $\varepsilon_{n+1}>0$ so that

$$
(1+\delta)^{-1} \varepsilon_{1},(1+\delta)^{-1} \varepsilon_{2}, \ldots,(1+\delta)^{-1} \varepsilon_{n}, \varepsilon_{n+1}
$$

is an admissible sequence for $\alpha$. Let $\varepsilon_{n+1}(\alpha)$ denote the supremum of possible values of $\varepsilon_{n+1}$ for a given $\alpha$.

By the transfinite induction argument of part (1) of this lemma, $\inf _{\alpha \in \Omega} \varepsilon_{n+1}(\alpha)$ $>0$.

Thus there is an $\varepsilon_{n+1}$ such that

$$
(1+\delta)^{-1} \varepsilon_{1}(1+\delta)^{-1} \varepsilon_{2},(1+\delta)^{-1} \varepsilon_{3}, \ldots,(1+\delta)^{-1} \varepsilon_{n}, \varepsilon_{n+1}
$$

is admissible for every $\alpha \in \Omega$. Thus Lemma 6 is proven.

Let $\left(\varepsilon_{i}\right)_{i=1}^{\infty}$ be a sequence such that $\left(\varepsilon_{i}\right)_{i=1}^{n}$ is admissible for each $n$ and $\alpha \in \Omega$. Assume each $\varepsilon_{i}<1$. Let $Z$ be the algebra of all analytic functions taking values in $A$, which are defined on a neighbourhood of $\sigma_{e}(x)$.

For each $g \in Z$, define

$$
\begin{aligned}
\|g\|^{(1)}=\inf \left\{\|c\|_{A}+\sum_{i=1}^{N} \varepsilon_{i}^{-1} \cdot \sup _{\lambda \in U_{i}}\left\|g_{i}(\lambda)\right\|_{A}:\right. \\
\left.\quad\left(\lambda \cdot 1_{A}-x\right) f(\lambda)+g(\lambda)=c+\sum_{i=1}^{N} g_{i}(\lambda)\left(\text { all } \lambda \in U_{N}\right)\right\}
\end{aligned}
$$

(this is a seminorm on $Z$ ); and

$$
\|g\|^{(2)}=\sup _{h \in Z,\|h\|^{(1)} \neq 0}\left(\|g h\|^{(1)} /\|h\|^{(1)}\right) .
$$


We shall see that $\|\cdot\|^{(2)}$ is also a seminorn on $Z$; it is always finite, and has the algebra norm property that $\left\|g_{1} g_{2}\right\|^{(2)} \leq\left\|g_{1}\right\|^{(2)} \cdot\left\|g_{2}\right\|^{(2)}$ for all $g_{1}, g_{2} \in Z$.

First, we claim that $A$ is isometrically embedded in $\left(Z,\|\cdot\|^{(2)}\right)$. For, by the definition of $\|\cdot\|^{(1)}$, we see that for all $c \in A, h \in Z$.

$$
\|c \cdot h\|^{(1)} \leq\|c\|_{A} \cdot\|h\|^{(1)}
$$

thus $\|c\|^{(2)} \leq\|c\|_{A}$. However,

$$
\begin{gathered}
\|c\|^{(1)}=\inf \left\{\|d\|_{A}+\sum_{i=1}^{N} \varepsilon_{i}^{-1} \cdot \sup _{\lambda \in U_{i}}\left\|g_{i}(\lambda)\right\|_{A}:\right. \\
\left.c=\left(\lambda \cdot 1_{A}-x\right) f(\lambda)+d+\sum_{i=1}^{N} g_{i}(\lambda)\left(\text { all } \lambda \in U_{N}\right)\right\} \\
\geq \inf \left\{\|d\|_{A}+\|c-d\|_{A}\right\}=\|c\|_{A} .
\end{gathered}
$$

since $\left(\varepsilon_{i}\right)_{i=1}^{N}$ is admissible for every $N$. So

$$
\|c\|^{(2)} \geq\left\|c \cdot 1_{A}\right\|^{(1)} /\left\|1_{A}\right\|^{(1)}=\|c\|_{A} ;
$$

therefore $A$ is indeed embedded isometrically. Next, we show that there is an inverse to $\mu \cdot 1_{A}-x$ in $\left(Z .\|\cdot\|^{(2)}\right)$ for all $\mu \notin \sigma_{e}(x)$; and to do this we must show that the function $R_{\mu}: \lambda \rightarrow(\mu-\lambda)^{-1} \cdot 1_{A}$ has finite norm $\left\|R_{\mu}\right\|^{(2)}$. But this is true of any function $g \in Z$. For if $g$ is defined on a neighbourhood of $\sigma_{e}(x)$, then for some $n$ it will be defined and bounded on $U_{n}$. Then, if $h \in Z$, let us say $\delta>0$ and

$$
h(\lambda)=(\lambda-x) k(\lambda)+c+\sum_{i=1}^{M} \varepsilon_{i}^{-1} \sup _{\lambda \in U_{i}}\left\|g_{i}(\lambda)\right\|_{A}-\delta
$$

with

$$
\|h\|^{(1)} \geq\|c\|_{A}+\sum_{i=1}^{M} \varepsilon_{i}^{-1} \sup _{\lambda \in U_{i}}\left\|g_{i}(\lambda)\right\|-\delta
$$

Then

$$
g h(\lambda)=(\lambda-x) g k(\lambda)+\sum_{i=1}^{M} g f_{i}(\lambda)+c g(\lambda) \quad\left(\lambda \in U_{m} \cap U_{n}\right)
$$

and

$$
\begin{aligned}
&\|g h\|^{(1)} \leq\left(\sup _{\lambda \in U_{n}}\|g(\lambda)\|\right) \cdot\left[\left(\|c\|+\sum_{i=1}^{n} \sup _{\lambda \in U_{2}}\left\|g_{i}(\lambda)\right\|\right) \cdot \varepsilon_{n}^{-1}\right. \\
&\left.+\sum_{i=n+1}^{M} \varepsilon_{i}^{-1} \sup _{\lambda \in U_{i}}\left\|g_{i}(\lambda)\right\|\right] \\
& \leq \varepsilon_{n}^{-1} \sup _{\lambda \in U_{n}}\|g(\lambda)\|\left(\|h\|^{(1)}+\delta\right) \quad\left(\text { for each } \varepsilon_{i} \leq 1\right) .
\end{aligned}
$$

Hence

$$
\|g\|^{(2)} \leq \varepsilon_{n}^{-1} \cdot \sup _{\lambda \in U_{n}}\|g(\lambda)\|
$$


The third remark we make about $\|\cdot\|^{(2)}$ is that is has the algebra norm property that

$$
\left\|g_{1} g_{2}\right\|^{(2)} \leq\left\|g_{1}\right\|^{(2)} \cdot\left\|g_{2}\right\|^{(2)}
$$

for all $g_{1}, g_{2} \in Z$.

For

$$
\begin{aligned}
\left\|g_{1}, g_{2}\right\|^{(2)}= & \sup _{\substack{h \in Z \\
\|h\|^{(1)} \neq 0}}\left(\frac{\left\|g_{1} g_{2} h\right\|^{(1)}}{\|h\|^{(1)}}\right) \\
= & \sup _{\substack{h \in Z \\
\|h\|^{(1)} \neq 0 \\
\left\|g_{2} h\right\|^{(1)} \neq 0}}\left(\frac{\left\|g_{1} g_{2} h\right\|^{(1)}}{\left\|q_{2} h\right\|^{(1)}} \cdot \frac{\left\|g_{2} h\right\|^{(1)}}{\|h\|^{(1)}}\right) .
\end{aligned}
$$

It is legitimate to restrict our attention to functions $h$ such that $\left\|g_{2} h\right\|^{(1)} \neq 0$ since if $\left\|g_{2} h\right\|^{(1)}=0$, then the expression $(*)$ is certainly zero for this function $h$; thus $\left\|g_{1} g_{2}\right\|^{(2)} \leq\left\|g_{1}\right\|^{(2)} \cdot\left\|g_{2}\right\|^{(2)}$.

Now, let $I$ be the ideal in $Z$ consisting of all functions $g$ whose seminorm $\|g\|^{(2)}$ is zero. Let $A^{\prime}$ denote the completion of the quotient space $Z / I$. Then $A^{\prime}$ is a Banach algebra in which $A$ is embedded isometrically, just as it is embedded isometrically in $\left(Z,\|\cdot\|^{(2)}\right)$. But for all $\mu \notin \sigma_{e}(x)$, there is an inverse for the element $\mu \cdot 1_{A}-x$ in $Z$, hence also in $A^{\prime}$. Thus $A^{\prime}$ is an extension of $A$ in which the spectrum of $x$ is precisely the essential spectrum of $x$ in $A$.

This concludes the proof of Theorem 1 .

ACKNOWLEDGEMENTS. The author would like to thank Dr. B. Bollobás for his help and encouragement during the preparation of this paper. This research was funded by a grant from the Science Research Council of the United Kingdom.

\section{REFERENCES}

1. G. E. Shilov, On normed rings with one generator, Mat. Sb. 21(63) (1947), 25-46.

2. Richard Arens, Linear topological division algebras, Bull. Amer. Math. Soc. 53 (1947), 623-630.

3. B. Bollobás, Adjoining inverses to Banach algebras, Trans. Amer. Math. Soc. 181 (1973), 165174.

4. __ Best possible bounds to the norms of inverses adjoined to normed algebras, Studia Math. 51 (1974), 87-96.

5. __ Adjoining inverses to commutative Banach algebras, Algebras in Analysis (J. H. Williamson, ed.), Academic Press, New York, 1975, pp. 256-257.

6. J. A. Lindberg, Extensions of algebra norms and applications. Studia Math. 40 (1971), 35-39.

Department of PuRe Mathematics and Mathematical Statistics, Cambridge UNIVERSITY, CAMBRIDGE, ENGLAND 